\title{
Consideraciones para el diseño del modulador y demodulador de la estación terrena CUBESAT-UD
}

\author{
Lilia E. Aparicio(1,2), Leonardo Plazas-Nossa(1) y Nestor J. Rodriguez ${ }^{(2)}$ \\ (1) Univ. Distrital Francisco José de Caldas, Facultad de Ingeniería, Maestría en Ciencias de la Información y las \\ Telecomunicaciones con énfasis en Teleinformática, Bogotá-Colombia. (correo-e: leap0763@gmail.com, \\ Iplazasn@udistrital.edu.co) \\ (2) Univ. Distrital Francisco José de Caldas, Grupo de Investigación GITEM++, Facultad de ingeniería, Bogotá-Colombia \\ (correo-e: ing.nestor0818@gmail.com)
}

Recibido Abr. 12, 2019; Aceptado Jun. 18, 2019; Versión final Sep. 3, 2019, Publicado Feb. 2020

\begin{abstract}
Resumen
En el presente artículo se analizan las distintas técnicas de modulación digital que se utilizan en los enlaces de comunicación desde una estación terrena hacia picosatélites que circundan la órbita LEO. La estación terrena Cubesat-UD, localizada en la ciudad de Bogotá, requiere establecer los enlaces de comunicación hacia la red SATNET (red satelital). Para ello es necesario establecer el diseño y el esquema de modulación más óptimo para su implementación, Por otra parte, se muestra una metodología propuesta para la implementación del modulador y demodulador que utilizará la estación terrena Cubesat-UD. Finalmente se muestra el resultado del análisis para este diseño. Los resultados muestran que el diseño del modulador y demodulador de la estación terrena Cubesat-UD sea FSK (Frequency Shift Keying), con el fin de garantizar la transmisión de la información y la conexión apropiada con otras estaciones terrenas.
\end{abstract}

Palabras clave: modulación; FSK; demodulación; picosatélites; estación terrena; Cubesat-UD

\section{Design considerations for the modulator and demodulator of the CUBESAT-UD earth station}

\begin{abstract}
This article discusses the different digital modulation techniques used in communication links from an earth station to picosatellites surrounding the LEO orbit. The Cubesat-UD earth station, located in the city of Bogota, requires the establishment of communication links to the SATNET network (satellite network). For this purpose, it is necessary to establish the design and the most optimal modulation scheme for its implementation. A methodology is proposed for the implementation of the modulator and demodulator to be used by the CubesatUD earth station. Finally, the result of the analysis for this design is shown. The results show that the modulator and demodulator design of the Cubesat-UD earth station must be FSK (Frequency Shift Keying), to guarantee the information transmission and appropriate connection with other earth stations.
\end{abstract}

Keywords: modulation; FSK; demodulation; picosatellites; earth station; Cubesat-UD 


\section{INTRODUCCIÓN}

Los avances en las telecomunicaciones, principalmente en las comunicaciones satelitales, permiten desarrollar temáticas de investigación como por ejemplo los módulos de comunicaciones para transmitir datos e información de un origen a un destino de una forma más eficiente, por lo tanto las estaciones terrenas son importantes en las comunicaciones satelitales y algunas universidades están creando sus propias versiones de estaciones terrenas para incluirlas con la red SATNET (satelital Network) con el propósito de realizar misiones espaciales (McNeill et al., 2017). Con el fin de realizar la transmisión de la señal RF desde la estación terrena hacia un picosatélite, es necesario un sistema de radiotransmisión digital en donde las señales de modulación y demodulación se conforman por medio de un pulso digital y se transmiten usando sistemas de modulación analógicas como AM (Modulación por Amplitud) o FM (Modulación por Frecuencia) en procesos de comunicación bidireccional (Xue et al., 2015), utilizando señales portadoras analógicas para la transmisión de la información.

Para el caso del sistema de transmisión de la estación terrena Cubesat-UD junto con el sistema de modulación (Gil y Aparicio, 2018), se requiere tener una señal modulada adecuada para que el canal de transmisión sea eficiente. Si el medio de transmisión es no guiado, el esquema de modulación debe superar varias barreras como por ejemplo la potencia utilizada para el envío de la frecuencia de transmisión, por esto la UIT (Unión Internacional de Telecomunicaciones) regula cual es la frecuencia de envío y recepción de las portadoras ajustadas para que las utilicen los radioaficionados y entidades con propósitos investigativos (Middlestead, 2017), por lo anterior, cuando una estación terrena desea hacer la comunicación con otra estación deben utilizar el mismo esquema de modulación, con el propósito de compartir información de telemetría, que entre estaciones terrenas sirven como referentes de investigación o de alertas tempranas.

La modulación que se utilice en la estación terrena Cubesat-UD debe superar las limitaciones por los equipos usados, es decir, que el esquema de modulación utilizado debe superar costos y sobre todo disponibilidad de hardware, restringido a frecuencias VHF (Very-High Frecuency) o UHF (Ultra-High Frecuency), por esta razón el esquema de modulación que se escoja para la estación terrena debe limitar el ancho de banda fraccionario, el cual relaciona el ancho de banda divido por la frecuencia central. Mientras el ancho de banda se encuentre más cerca a la frecuencia central se minimizan los costos por los dispositivos utilizados (Zhou et al., 2016).

En otra instancia, la estación terrena Cubesat-UD de la Universidad Distrital Francisco José de Caldas y el grupo de investigación GITEM++ (Grupo de Investigación en Telemedicina) debe seleccionar un método de modulación que combata el ruido e interferencia (Aparicio et al., 2017), para ello el grupo de investigación establece la necesidad de encontrar el esquema de modulación que mejor se adapte en el proceso de comunicación con otras estaciones terrenas a nivel mundial, según resultados preliminares de diseños anteriores, se clasifica por tipo de producto, resultados obtenidos y factores de diseño, luego de este proceso se obtiene los parámetros de diseño del sistema modulador y demodulador específico para la estación terrena mediante el esquema de modulación FSK (Frequency Shift Keying) para la comunicación con redes satelitales como lo por ejemplo SATNET (Kahn, 2017).

\section{EL MODELO}

El modelo propuesto para el diseño del modulador y demodulador que se implementará en la estación terrena Cubesat-UD, tendrá la característica principal de realizar el proceso de modulación en esquema FSK por los resultados obtenidos en la sección de resultados y discusión, para la transmisión de la información como se muestra en la Fig. 1, se relaciona la entrada de información de datos como una fuente $X$ de estados binarios que contendrá información de misión, telemetría etc., por medio del uso del protocolo de transmisión AX.25 estándar para las comunicaciones satelitales, que organiza los bits hacia el modulador diseñado y se realiza la transmisión de la información en FM (frecuencia modulada) por medio de la antena (Aparicio, 2016).

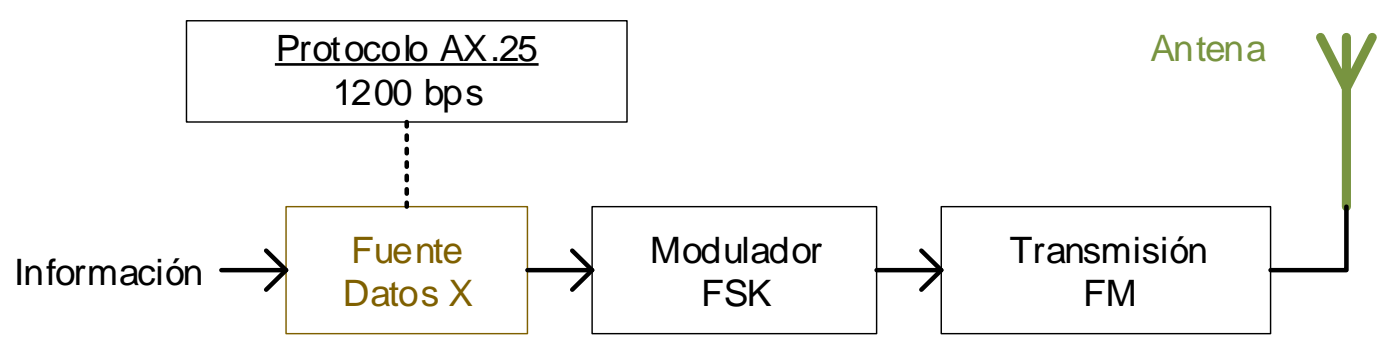

Fig. 1: Esquema de transmisión de la información. Fuente Autor. 
Para la recepción de la información en la estación terrena Cubesat - UD, se plantea el modelo de recepción como se muestra en la Fig. 2, este realiza el proceso inverso al de transmisión puesto que se captura la señal por medio de la antena y en frecuencia FM, luego demodula la señal utilizando FSK y se obtienen los datos binarios que se han obtenido organizados según el protocolo AX.25 (Aparicio, 2014).

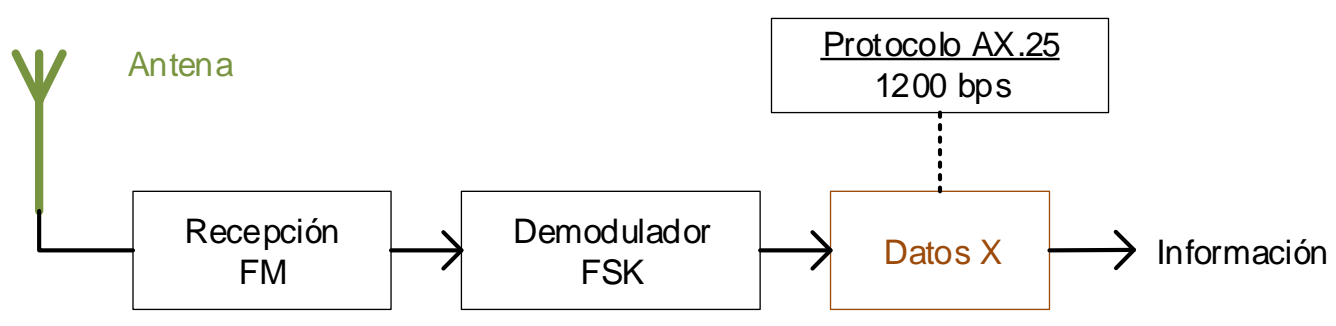

Fig. 2: Esquema de recepción de la información. Fuente Autor.

El modelo propuesto del modulador se presenta en la Fig. 3, este diseño se realiza por medio de descripción de hardware y uso de lenguaje VHDL (Very High Description Language) en él, se establecen los bit de entrada como datos binarios representados como 1 y 0 , que son llevados al módulo de contador descendente el cual tiene como función organizar estos en un vector de entraba ubicando los bits del menos significativo al más significativo, el siguiente proceso es el módulo selector de fase encargado de identificar si los bits ingresados presentan una fase alta o baja, y por último teniendo en cuenta la fase se crean las frecuencias de marca y espacio, para luego ser transmitidas por el sistema de transmisión FM.

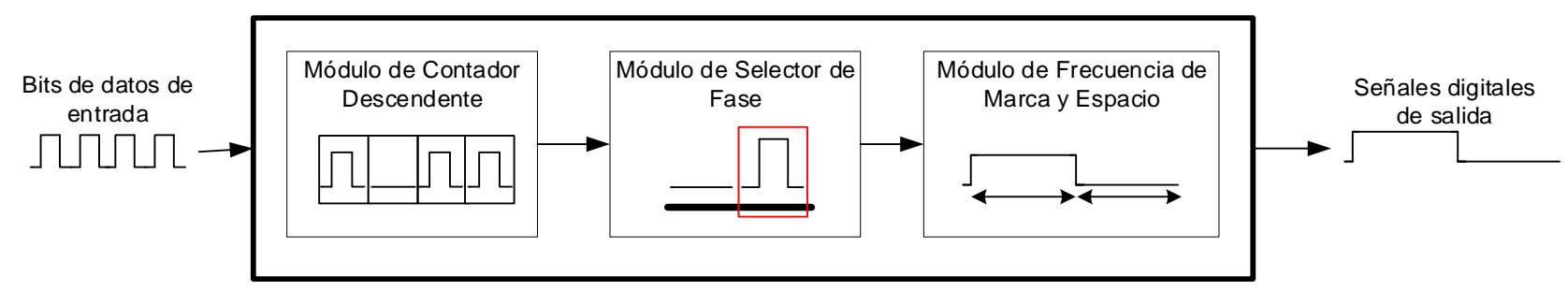

Fig. 3. Diseño del esquema de modulación propuesto. Fuente Autor.

El modelo propuesto para el demodulador presentado en la Fig. 4, muestra el proceso opuesto del modulador, en este, se recibe la señal digital y se lleva al módulo demodulador de frecuencia de marca y espacio que compara el estado de la señal para asignar un bit de frecuencia alto o bajo, una vez se tienen estos dígitos se organizan en el módulo de procesamiento de datos y estados que son incluidos en el vector de posición desde el valor menos significativo al más significativo obteniendo la trama de bits enviada.

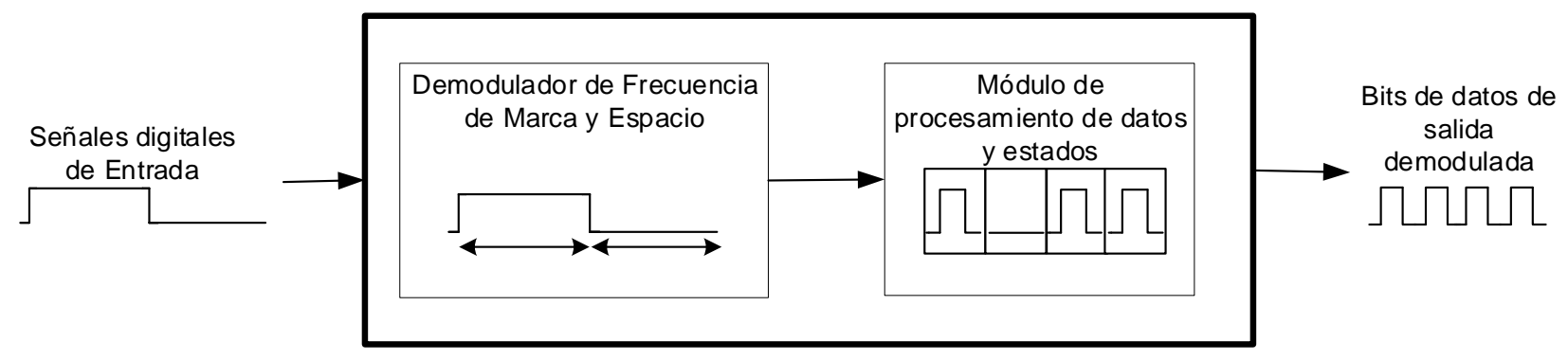

Fig. 4. Diseño del esquema de demodulación propuesto. Fuente Autor.

\section{ANÁLISIS DE ESQUEMAS DE MODULACIÓN}

Realizando una exhaustiva investigación respecto a estaciones terrenas y picosatélites que se encuentran a nivel internacional, se obtiene algunos tipos de picosatélites, junto con sus frecuencias de subida y bajada para la transmisión de la información y el tipo de esquema de modulación que utiliza, entre las cuales se puede encontrar: GMSK, QPSK, SSB/CW, BPSK, AFSK y FSK, es de tener en cuenta que el esquema de modulación AFSK es una variación del esquema de modulación FSK que representa modulación por desplazamiento de frecuencia de audio. La Fig. 5 permite el análisis y revisión del tipo de esquemas de modulación utilizadas para la transmisión de señales, para ello se toman como base 22 picosatelites y nanosatélites que tienen proyectadas misiones de operación, teniendo en cuenta la base de datos de AMSAT-EA hasta el año 2018 (AMateur SATélite - Asociación mundial de satélites de radioaficionados) dando como resultado que el $54.5 \%$ de estos picosatélites utilizan la 
modulación FSK para la transmisión de los datos, lo que implica que un gran número de estos dispositivos permiten la interconexión de enlaces y datos con modulación digital, por ende la estación terrena tendrá una mayor extensión de estaciones terrenas y picosatélites a las cuales pueda conectarse y compartir información como datos de telemetría (Aparicio, 2013).

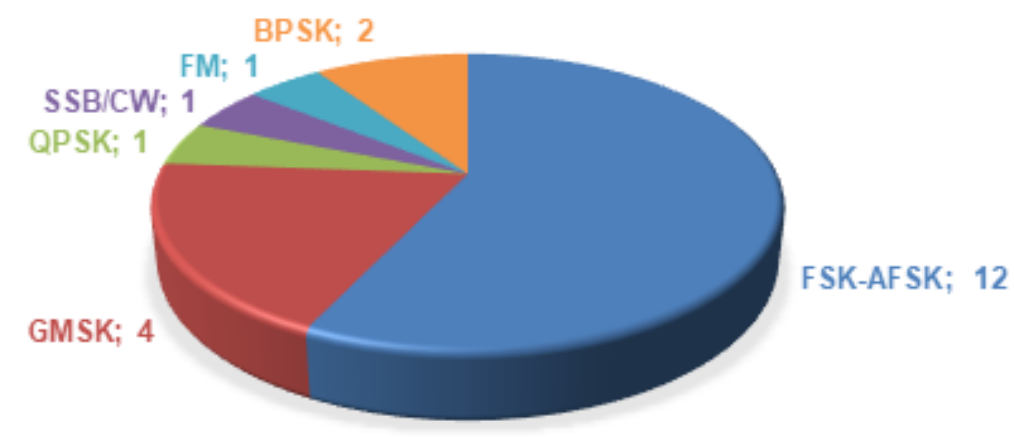

Fig. 5. Esquemas de modulación de picosatélites internacionales. Fuente Autor.

Los esquemas de modulación que han sido utilizados en la implementación y puesta en marcha de la estación terrena Cubesat-UD, permiten el análisis e identificación de las características principales para la comunicación con los pico-satélites, por ende, en esta investigación se presenta los resultados obtenidos de esquemas de modulación por medio de simulaciones utilizando el software MATLAB, en estos se puede observar de forma virtual el comportamiento de los esquemas de modulación, análisis de los diagrama de fases y constelaciones utilizando: 8-PSK, 16-PSK, 4-QAM, 8-QAM o 16-QAM, esquemas de modulación que aunque bajan la tasa de bit respecto a los bits de entrada, en la práctica su uso no es común en comunicaciones satelitales (Tsai et al., 2017).

En la Fig. 6, se presentan los esquemas de modulación en los cuales se han trabajado como productos de investigación para el diseño, análisis e implementación para la estación terrena Cubesat-UD datos obtenidos hasta el año 2017, producción desarrollada por grupo de investigación GITEM++ con un total de 45 productos, resulta interesante analizar que el esquema de modulación más pertinente para la estación terrena Cubesat-UD según estos productos es FSK con el $36 \%$ de la totalidad de resultados obtenidos, apoyándose en aspectos tanto teóricos como investigaciones en proceso de comunicaciones, estos productos también son el resultado de desarrollar e innovar en el diseño de la estación terrena utilizando especificaciones de conexión establecidas por redes satelitales internacionales, las cuales definían como uso obligatorio el esquema de modulación FSK dadas sus ventajas de implementación de bajo costo, puesto que tiene cierta afinidad con esquemas de modulación analógico como FM (Ferro y Espindola, 2013) que modula mediante el cambio de frecuencia de la señal portadora a fin de obtener un ancho de banda más amplio, suficiente para la transmisión de la información que la estación terrena Cubesat-UD necesita (Adnan et al., 2016).

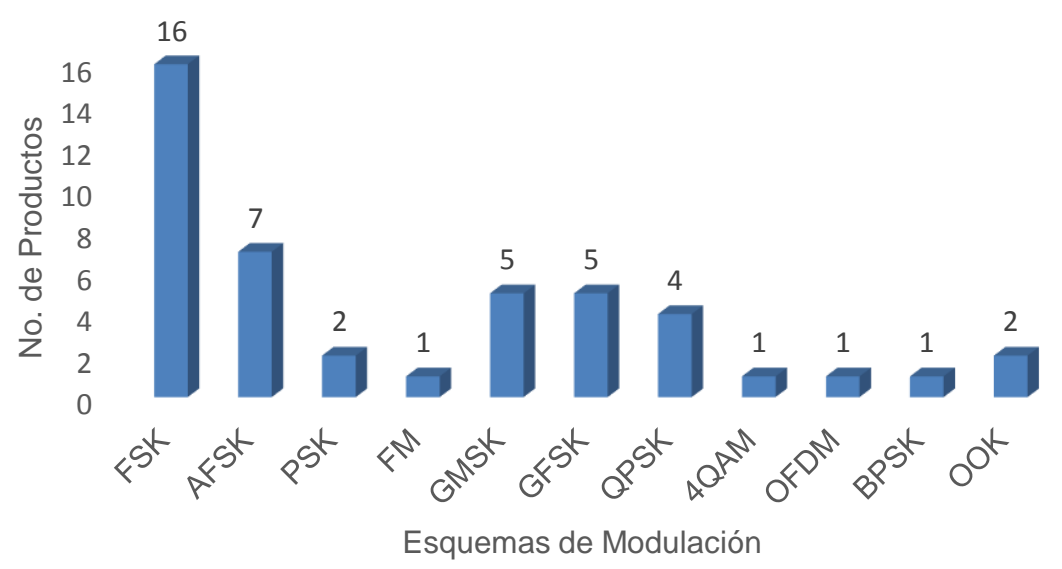

Fig. 6. Esquemas de modulación utilizados en los diseños de la estación terrena. Fuente Autor.

\section{RESULTADOS Y DISCUSIÓN}

El módulo de comunicaciones diseñado para el picosatélite Cubesat-UD por parte de los investigadores de la Universidad Distrital Francisco José de Caldas, establece parámetros de diseño tales como el monitoreo de la recepción de señales provenientes de la estación terrena, función de informar al OBC y al submódulo de Beacon cuando se reciban datos, recepción de señales RF y conversión a señales de frecuencia intermedia para su 
demodulación, el cual se utiliza FSK a una tasa de bit de 1200 o 9600bps, uso del protocolo AX.25 para la recepción de la trama de datos, en la Fig. 7 se puede observar el diseño en diagrama de bloques del módulo de comunicaciones, en el cual los investigadores tienen en cuenta el uso del esquema de modulación FSK abierto al uso internacional.

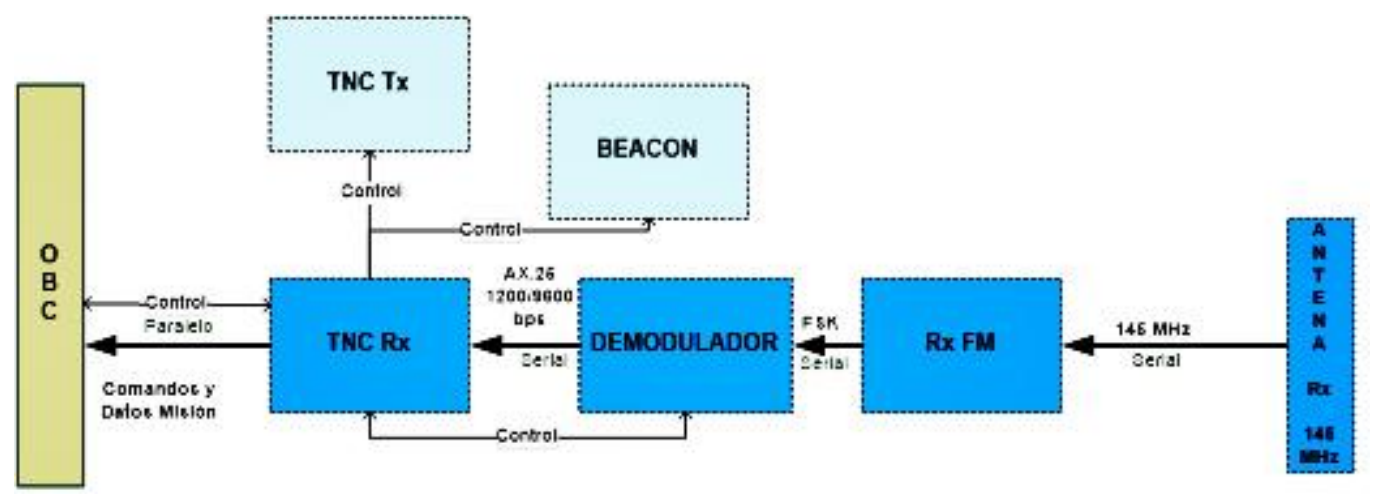

Fig. 7. Diseño en diagrama de bloques del módulo de comunicaciones. (Reproducida con autorización de Aparicio, 2017)

El diagrama de bloques representa el diseño crítico del módulo de comunicaciones del picosatélite, en él, se resalta el uso de equipos de comunicación como el Radio FM (Yaesu VX-1R y Alinco DJ-C5T), antenas tipo dipolo para recepción a frecuencia de $145 \mathrm{MHz}$, modem MX614 y uso de microcontroladores para realizar la función de TNC (Terminal Node Controler), uso de demodulador a una taza de bit de transmisión de 1200bps o 9600bps con esquema de modulación FSK, lo que permite concluir que los investigadores y diseñadores del sistema de comunicaciones establecen como mejor opción la modulación FSK, por sus aplicabilidad y diseño (Plazas-Nossa y Segura, 2012). Es de tener en cuenta que estos equipos y diseño son parte de proyectos de investigación para acceder a la financiación por parte de la Universidad Distrital Francisco José de Caldas, por lo que cada uno de los segmentos se diseñó por secciones con la tecnología proporcionada y cuyo objetivo final es la realización de un laboratorio físico de experimentación para estudiantes de telecomunicaciones.

La Fig. 8 presenta el diseño del diagrama de bloques para el módulo de comunicaciones del picosatélite, esta vez para la frecuencia de bajada en comunicación con la estación terrena, en este diagrama se puede observar los siguientes elementos que son comunes en el diseño del dispositivo, un Radio FM (Yaesu VX-1R y Alinco DJC5T), antenas tipo dipolo para recepción a frecuencia de $435 \mathrm{MHz}$, modem MX614 y Microcontroladores para funciones de TNC, el diagrama vuelve a mostrar que los diseñadores utilizan el modulador para la transmisión de los datos a una tasa de bit nombrada anteriormente, el esquema de modulación escogido para el diseño es FSK, lo que ratifica el uso de este esquema de modulación (Zhou et al., 2016).

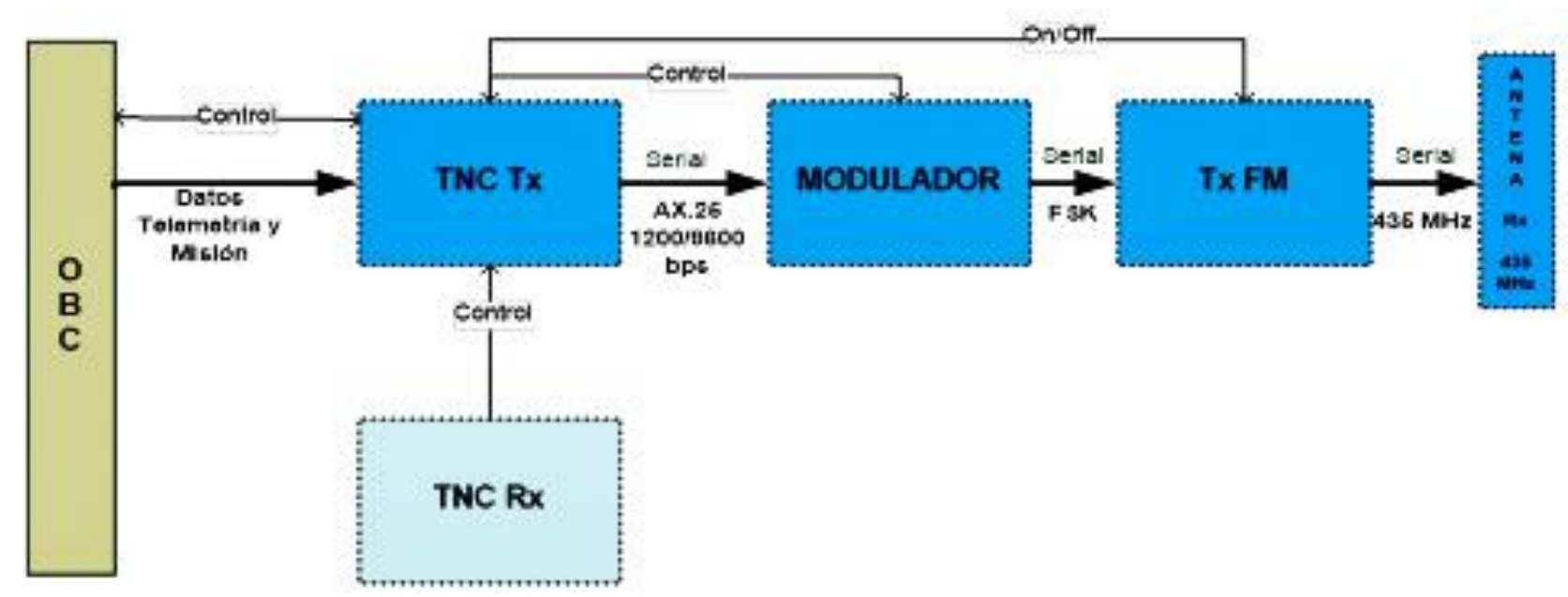

Fig. 8. Diseño del diagrama de bloques para el módulo de comunicaciones del pico-satélite. (Reproducida con autorización de Aparicio, 2017).

Por otra parte, los investigadores realizan el diseño de simulación del enlace de comunicación desde el transmisor de la estación terrena, junto con la simulación del medio por medio de las pérdidas del canal hasta llegar al receptor satelital, como se puede apreciar en la Fig. 9, en la cual se realiza una modulación por medio de modulación y demodulación M-FSK, fuente de datos binaria, codificador convolucional, pérdidas de canal, decodificador Viterbi, entre otros. 


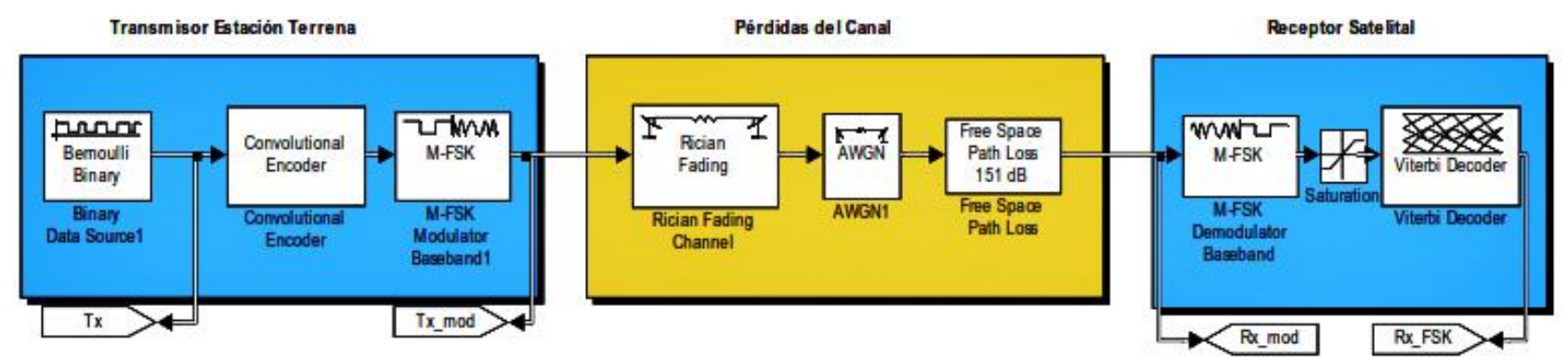

Fig. 9. Diseño del enlace satelital de comunicaciones mediante modulación M-FSK. (tomada de Aparicio, 2017).

Luego de realizar el análisis de la simulación del enlace de comunicaciones utilizando esquema de modulación M-FSK, se obtiene la Fig. 10, en la cual se presenta la relación de la señal a ruido contra el BER (Bit Error Rate) aplicando parámetros de simulación como el modelo semi-analítico de Monte Carlo, por lo que la figura demuestra robustez en el enlace, por ejemplo si se considera una señal con relación señal a ruido de $24 \mathrm{~dB}$ se obtiene un BER de $1 e^{-6}$. Entre estos resultados se mencionan los desarrollados por Arthur y Allen (2015).

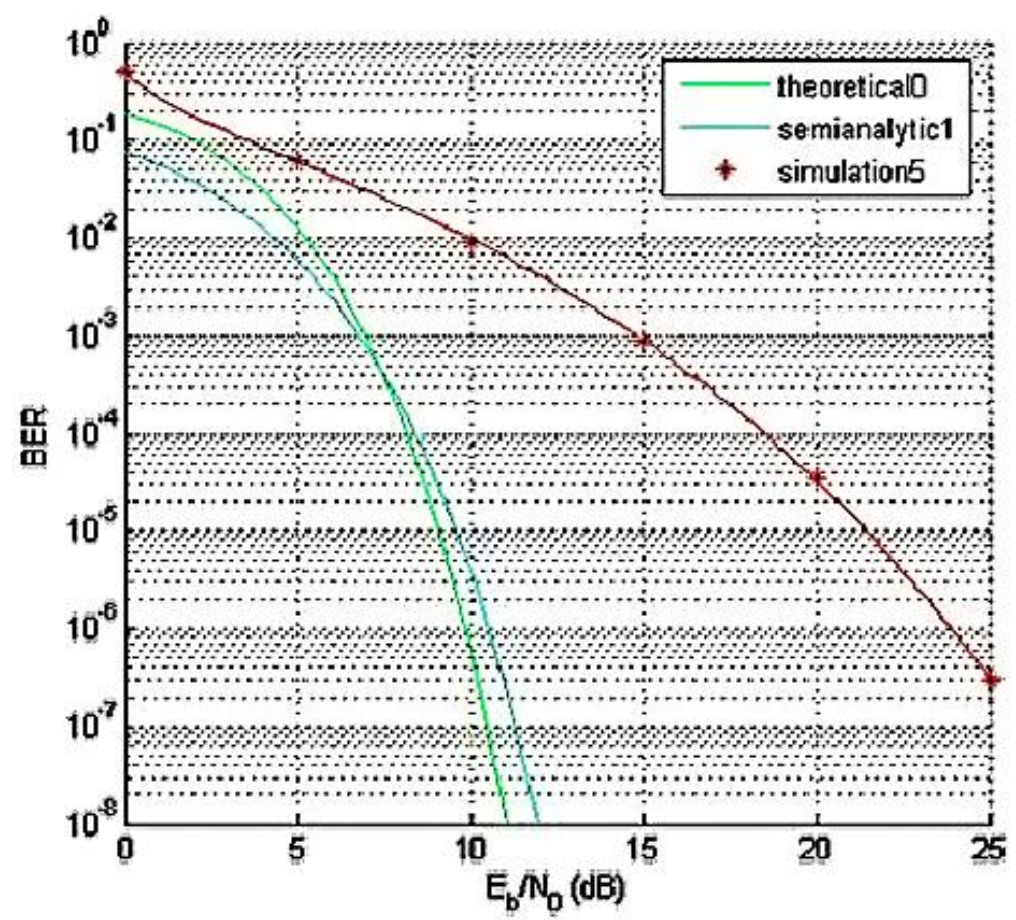

Fig. 10. Relación de señal a ruido vs BER. (Reproducida con autorización de Garay \& Florez, 2008)

Otra forma de contribuir a los esquemas de modulación lo exhibe la universidad Sur Colombiana (USCO) con el diseño de su estación terrena sede Neiva, esta muestra el análisis y desarrollo de distintos tipos de esquemas de modulación por medio de simulaciones como QPSK, 16QAM, 4QAM, 8FSK y GMSK relacionando en cada caso la energía de bit versus densidad de ruido (Eb/No), el resultado bajo un modelo diseñado en MATLAB se ha simulado el comportamiento del canal con modulación QPSK, Ruido Blanco Gaussiano, perdidas por espacio libre y dispersión Rician, algunos ejemplos de optimización se presenta por (Gao et al., 2017; Liu et al., 2017), encontrando así los índices que determinan cuales esquemas de modulación son aptos para el desarrollo del sistema de comunicaciones de la estación terrena, algunos de estos lo menciona (Shamee et al., 2010). Entre los resultados obtenidos por parte de los investigadores en entornos ideales como lo son las simulaciones en el software de MATLAB, se puede analizar las pruebas resultantes, en las cuales se observan que los esquemas de modulación que tienen una menor pérdida de tasa de bits son: QPSK, 4-QAM y GMSK debido a la observación de los diferentes valores de energía de bit a densidad de ruido Eb/No, frente a un BER deseado. Por otra parte, los resultados muestran que la modulación GMSK presenta una modulación apta para la estación terrena sede Neiva dado que permite disminuir la interferencia Inter símbolo.

Además de la universidad USCO, existen otras universidades como la Universidad Pedagógica y Tecnológica de Colombia que también desarrolló su propia estación terrena, ellos diseñaron esta estación con el objetivo de hacer seguimiento de picosatélites en órbita LEO. El desarrollo del sistema de comunicaciones se realizó por medio de fases las cuales se implementaron de la siguiente forma: para la fase I se realiza la caracterizaron del canal de 
comunicaciones para encontrar pérdidas de enlace y parámetros de este, en la Fase II se identifican las características tecnológicas y módulos del sistema, en la Fase III determinan las características de los equipos de comunicación y para la Fase IV definen las normativas de transmisión de los equipos (Aparicio, 2012).

En esta investigación se observan los resultados obtenidos a partir de la Fase III y IV donde definen los equipos que están utilizando y su respectivo esquema de modulación, para ello el grupo de investigación utiliza el transceptor TM-D700 con el que logran realizar capturas de señal de los picosatélites AO-51 y AO-27 demostrando así que el uso de este dispositivo permite la comunicación mediante la modulación FSK y les garantiza tener el enlace desde la estación terrena hacia el picosatélite.

Otros resultados obtenidos corresponden realizar la estimación de la velocidad efectiva de transmisión junto con los equipos de la estación terrena y el esquema de modulación FSK, como se puede apreciar en la Fig. 11, los resultados presentan una velocidad de transmisión vs el número de tramas enviadas (k) a 1200 bps utilizando el protocolo AX.25, dando como resultado que la eficiencia de la velocidad de transmisión para un máximo de 7 tramas es de 1110,19 bps. En conclusión, se deduce que el uso del esquema de modulación FSK tiene un resultado óptimo para realizar el envío de las tramas de información, por otra parte, se identifican resultados obtenidos por Szczepaniak y Piotrowskique (2017) ratifica el esquema de modulación seleccionado.

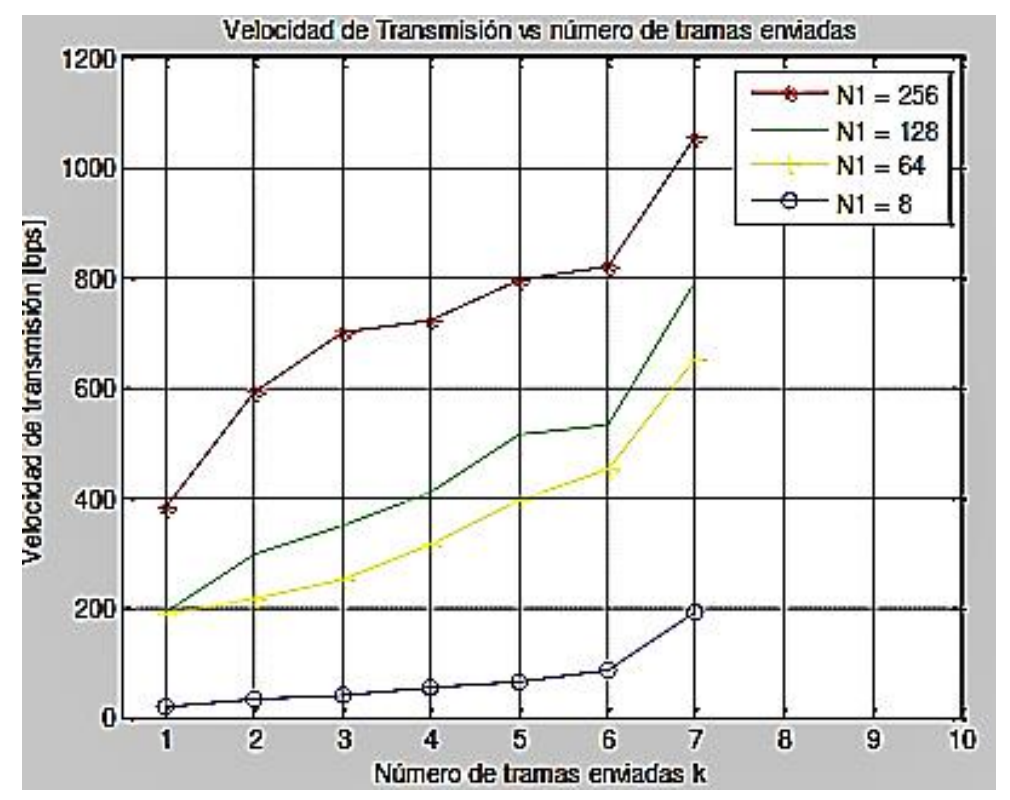

Fig. 11. Velocidad de transmisión vs número de tramas enviadas a 1200 bps.

(Reproducida con autorización de Maria, 2015)

\section{CONCLUSIONES}

A partir de los resultados obtenidos, se pueden extraer las siguientes conclusiones: 1) La universidad Sur Colombiana encontró que dentro de los esquemas de modulación con los que pudo hacer contacto con picosatélites fue FSK dando el resultado esperado por medio de la estación terrena 2) La Universidad Pedagógica y Tecnológica de Colombia encontró dentro de sus resultados de simulación que el esquema de modulación digital FSK que se encuentra en los equipos físicos de la estación terrena permite la comunicación con los picosatélites que se encontraron en la órbita LEO 3). De los análisis realizados anteriormente, se puede concluir que el esquema de modulación FSK tiene un buen rendimiento para la operabilidad de la estación terrena dado que las pérdidas de espacio libre que se han obtenido físicamente no superan los $135.68 \mathrm{~dB}$ de subida y $145.22 \mathrm{~dB}$ de bajada a una distancia de $800 \mathrm{Km}$, por lo que se tendrá una atenuación por kilómetro recorrido de $2.887 e^{-3} \mathrm{~dB} / \mathrm{Km}$ con una potencia de señal transmitida por la antena de $30.4 \mathrm{~W}$, haciendo que la tasa de transmisión de bits sea de $51200 \mathrm{Kbps}$. Las ventajas del uso de la modulación FSK es que la implementación física es más asequible respecto a otras técnicas de modulación, además genera un bajo costo para la operación de la estación terrena Cubesat UD y una mayor inmunidad a las interferencias que se encuentren a su alrededor. 4). La modulación FSK tiene una mayor resistencia al ruido e interferencia a través de frecuencias UHF, la estación terrena Cubesat UD utiliza frecuencias de $146 \mathrm{MHz}$ de enlace de subida y $438 \mathrm{MHz}$ de enlace de bajada transmitidas por una antena Yagi cruzada de $5.5 \mathrm{~m}$ con una ganancia de $16 \mathrm{~dB}$, lo que permite observar que las pérdidas de señal no superan los $0.5 \mathrm{~dB}$ de alimentación teniendo en cuenta que la energía de bit respecto al a densidad de potencia de ruido Eb/No es igual a $10.51 \mathrm{~dB}$. Utilizando una modulación 8-FSK se analiza que el BER no es superior a $10 e^{-6}$ por lo que la tasa de pérdidas 
de bits es baja, si se utilizan frecuencias que no superen los $450 \mathrm{Mhz}$. 5). Es de vital importancia que el diseño del modulador y demodulador de la estación terrena Cubesat-UD sea FSK para garantizar la transmisión de la información y la conexión con otras estaciones terrenas.

\section{REFERENCIAS}

Adnan, B. M. y Alamgir, F. M., Performance simulation and comparison in High Altitude Platforms (HAPs) communications systems under PSK, DPSK, QAM \& FSK modulation schemes and AWGN, Rician \& Rayleigh communication channels, DOI: 10.1109/IEMCON.2016.7746080, IEEE 7th Annual Information Technology, Electronics and Mobile Communication Conference (IEMCON), Vancouver, BC, pp. 1-11. (2016)

Aparicio, L., Cubeas-UD Telemedicine and telemetry technical training, Memorias Primer Congreso Internacional De Ciencia Y Tecnologia Aeroespacial, ISSN: 2011-7760, 2(5), 30 -33, (2012)

Aparicio, L., Design and simulation of an attitude determination system based on the Extended Kalman Filter for Cube-Sat Colombia I, Revista Facultad de Ingeniería, ISSN: 0120-6230, (7), 146 - 154, (2014)

Aparicio, L., Guía de pruebas y protocolos para el lanzamiento del satélite Colombia I, Redes De Ingeniería, ISSN: 2248-762X, 68-73, (2016)

Aparicio, L., Oyola, J. y Rodriguez, J., Designs and implementation for CUBESAT COLOMBIA I satellite power module, International Journal of Applied Engineering Research, ISSN: 0976-4259, 12(18) 7360 - 7371 (2017)

Aparicio, L., Subchasis de integración modular para el despliegue de antenas en un cubesat, Tecnura, ISSN: 0123-921X, (17), $149-160,(2013)$

Arthur, A., Giordano, A. y Levesque, H., Digital Communications BER Performance in AWGN (FSK and MSK), in Modeling of Digital Communication Systems Using SIMULINK, DOI: 0.1002/9781119009511.ch5, Wiley Telecom, 1, pp.416, (2015)

Ferro, R. y Espindola, J. Direccionamiento automático de antenas en estaciones terrenas de seguimiento a picosatelites, Tecnura, ISSN: 0123-921X, 17(35), 26 - 37, (2013)

Gao, H., y otros cuatro autores, Additive phase noise suppression of direct modulation analog optical links, DOI: 10.1109/APCAP.2017.8420745, Sixth Asia-Pacific Conference on Antennas and Propagation (APCAP), Xi'an, China, pp. 1-3. (2017)

Gil, A. y Aparicio, L., Setting of an earth satellite station in The District University Francisco Jose De Caldas, DOI: http://dx.doi.org/10.17654/EC018030407, Far East Journal of Electronics and Communications, Brazil, 18( 3), 407 - 418, (2018)

Kahn, R. Introduction of Packet Satellite Communications, NTC Conference Record - National Telecommunications Conference, Washington, DC, USA, pp. 45. 1. 1-45. 1. 8. (2017)

Liu, Z., Liu, D., Zhang, J. y Liu, J., Modulation and demodulation circuits of MIMO radar based on qpsk, DOI: 10.1109/APCAP.2017.8420619, Sixth Asia-Pacific Conference on Antennas and Propagation (APCAP), Xi'an, China, pp. 1-3. (2017)

McNeill, D. y otros siete autores, Satnet Monitoring and Control, NTC Conference Record - National Telecommunications Conference, Washington, DC, USA, pp. 45. 3. 1-45. 3. 4. (2017)

Middlestead R. W., Frequency Shift Keying (FSK) Modulation, Demodulation, and performance, in Digital Communications with Emphasis on Data Modems:Theory, Analysis, Design, Simulation, Testing, and Applications, DOI: 10.1002/9781119011866.ch5, Wiley Telecom, 1, pp.824, (2017)

Plazas-Nossa, L. y Segura, D., Algoritmo para la corrección sin puntos de referencia del fenómeno de perspectiva para sistema de captura de imágenes del CubeSat UD Colombia II, Memorias Primer Congreso Internacional De Ciencia Y Tecnología Aeroespacial, ISSN: 2011-7760, 1 - 11, (2012)

Shamee, B. y otros cuatro autores, GauSsian Minimum Shift Keying for spectrally efficient and dispersion tolerant optical communications, DOI: 10.1364/CLEO.2010.JThE54, CLEO/QELS: Laser Science to Photonic Applications, San Jose, CA, pp. 1-2, (2010)

Szczepaniak, E. and Piotrowski, Z., Radio transmission masking on the example of FSK modulation, DOI: 10.23919/SPA.2017.8166899, Signal Processing: Algorithms, Architectures, Arrangements, and Applications (SPA), Poznan, pp. 394-399. (2017)

Tsai, K., y otros cuatro autores, Gaussian minimum shift keying modulator [for satellite communication], DOI: 10.1109/AERO.2000.878495, IEEE Aerospace Conference. Proceedings (Cat. No.00TH8484), Big Sky, MT, vol.5, pp. 235241, (2000)

Xue, L., Wang, S., Li, M. and Wang, L., FM-to-AM conversion comparison of typical second-order phase modulation signals, DOI: 10.1109/ICIST.2015.7289032, 5th International Conference on Information Science and Technology (ICIST), Changsha, pp. 547-552. (2015)

Zhou, R., Zhang, Y., Rhee, W. y Wang, Z., 2.4GHz 20Mb/s FSK receiver front-end and transmitter modulation PLL design for energy-efficient short-range communicaiton, DOI: 10.1109/EDSSC.2016.7785265, IEEE International Conference on

Electron Devices and Solid-State Circuits (EDSSC), Hong Kong, pp. 291-294. (2016). 